\title{
Electrolyzed-reduced water increases resistance to oxidative stress, fertility, and lifespan via insulin/IGF-1-like signal in C. elegans
}

\author{
Seul-Ki Park ${ }^{1}$ and Sang-Kyu Park ${ }^{*}$ \\ ${ }^{1}$ Department of Medical Biotechnology, College of Medical Sciences, Soonchunhyang University, Asan, Chungnam, Republic of Korea.
}

\begin{abstract}
Electrolyzed-reduced water (ERW) scavenges reactive oxygen species and is a powerful anti-oxidant. A positive correlation between oxidative stress and aging has been proved in many model organisms. In Caenorhabditis elegans, many long-lived mutants showed reduced fertility as a trade off against longevity phenotype. We aimed to study the effect of ERW on oxidative stress, fertility and lifespan of $C$. elegans. We also investigated the genetic pathway involved in the effect of ERW on resistance to oxidative stress and lifespan. We compared lifespan and fertility of worms in media prepared with distilled water and ERW. ERW significantly extended lifespan and increased the number of progeny produced. Then the effect of ERW on resistance to oxidative stress and lifespan of long-lived mutants was determined. ERW increased resistance to oxidative stress and lifespan of eat-2, a genetic model of dietary restriction, but had no effect on those of age-1, which is involved in insulin/insulin-like growth factor (IGF)-1-like signal. In addition, knockdown of daf-16, the downstream mediator of insulin/IGF-1-like signal, completely prevented the effect of ERW on lifespan. These findings suggest that ERW can extend lifespan without accompanying reduced fertility and modulate resistance to oxidative stress and lifespan via insulin/IGF-1-like signal in C. elegans.
\end{abstract}

Key words: Electrolyzed-reduced water, aging, daf-16, fertility, lifespan.

\section{INTRODUCTION}

An electric current passed through water results in electrolysis, which produces electrolyzed-reduced water (ERW) near the cathode and electrolyzed-oxidized water near the anode. ERW has a negative redox potential and low oxidationreduction potential due to increased $\mathrm{pH}$, whereas electrolyzedoxidized water has a low $\mathrm{pH}$ and high oxidation-reduction potential (Shirahata et al., 1997). The most well-known bioactivity of electrolyzed-oxidized water is anti-bacterial activity. Electrolyzed-oxidized water can inactivate numerous microorganisms, including Salmonella enteritidis, Escherichia coli O157:H7, Listeria monocytogenes, and Campylobacter jejuni (Kim et al., 2000; Park et al., 002a; Venkitanarayanan et al., 1999). The effects of electrolyzed-oxidized water on microbial decontamination of food and medical disinfection have also been reported recently (Park et al., 2002b). ERW effectively scavenges reactive oxygen species (ROS) produced as a byproduct of normal cellular metabolism (Shirahata et al., 1997). Cellular ROS include the hydroxyl radical $\left(\mathrm{HO}^{*}\right)$, the superoxide radical anion $\left(\mathrm{O}_{2}{ }^{-}\right)$, nitric oxide $(\mathrm{NO})$, peroxyl radicals $\left(\mathrm{ROO}^{\circ}\right)$, peroxynitrite $\left(\mathrm{ONOO}^{-}\right)$, singlet oxygen $\left({ }^{1} \mathrm{O}_{2}\right)$, hypochlorous acid $(\mathrm{HOCl})$ and hydrogen peroxide $\left(\mathrm{H}_{2} \mathrm{O}_{2}\right)$ (Storz and Imlay, 1999). Since ROS can cause damage to virtually all cellular macromolecules, such as DNA, proteins and lipids, they are considered a major causal factor of many pathophysiological alterations. ROS-induced DNA damage and RNA degradation are significantly inhibited by ERW in vitro (Lee et al., 2006). ERW also prevents oxidative cleavage of cellular proteins in human lymphocytes (Lee et al., 2006). Recent studies have shown the therapeutic effect of ERW including the prevention of alloxan-induced type 1 diabetes mellitus and reduced carbon tetrachloride-induced liver damage (Li et al., 2001). ERW also shows positive effects on the local immune response in mice (Lee et al., 2009).

It has been hypothesized that ROS play a key role in normal aging of tissues that rely heavily on oxidative phosphorylation (Beckman and Ames, 1998; Sohal and Weindruch, 1996). The free radical theory of aging proposes that normal aging results from random deleterious damage to tissues caused by ROS (Sohal and Weindruch, 1996). Due to the potential role of antioxidants in modulating oxidative stress associated with aging, dietary interventions of ROSscavenging antioxidants have received particular attention. Resveratrol, a polyphenol found in red wine, significantly increases the lifespan of C. elegans and Drosophila melanogaster (Wood et al., 2004). Gene expression profiling has revealed that vitamin E supplementation can reverse several age-related changes in gene expression in the mouse heart and brain (Park et al., 2008). Curcumin, an Indian spice, protects brain from oxidative stress and scavenges nitric oxide-based radicals (Lakshmanan et al., 2011; Sreejayan and Rao, 1996). One study identified tissue-specific transcriptional biomarkers of aging through genome-wide transcriptome analysis and showed that supplementation of antioxidants significantly prevents age-related transcriptional changes of biomarkers of aging in a tissue-specific way in mice (Park et al., 2009). Interestingly, Yan et al. reported that C. elegans grown in water medium prepared with ERW live longer than worms grown in distilled water and have increased resistance to oxidative stress due to the ROS scavenging activity of ERW (Yan et al., 2010).

In C. elegans, the insulin/IGF-1-like signal activates the cellsurface receptor DAF-2. The signal is transferred to a PI3 kinase AGE-1 and AKT-1/AKT-2. The first lifespan-extending genetic mutant in C. elegans was the age-1 mutant (Johnson, 1990). Mutations in the genes involved in insulin/IGF-1-like signal 
increase lifespan significantly, suggesting reduced insulin/ IGF-1-like signal can extend lifespan in C. elegans (Johnson, 1990; Gems et al., 1998; Kenyon et al., 1993). Lifespan extension through reduced insulin/IGF-1-like signal is mediated by DAF16. DAF-16 is a transcription factor localized in the nucleus by reduced insulin/IGF-1-like signal and regulates the expression of many age-related genes (Tatar et al., 2003).

C. elegans is a free living nematode whose genome was fully sequenced in 1998 (The C. elegans Sequencing Consortium, 1998). The life cycle of C. elegans is relatively short. After hatching they develop through four larval stages, from L1 to L4. Adult hermaphrodite worms produce 300 progeny on average during their gravid period using self-fertilization. The average lifespan of $C$. elegans is about 15-17 days and a generation is about 4 days, which makes this animal as a good experimental organism for aging research (Wood, 1988).

We have reported that C. elegans grown in ERW show increased resistance to various environmental stressors, including oxidative stress, heat stress, and ultraviolet irradiation (Park et al., 2012). The aims of the study are elucidation of the effects of ERW on aging and longevity of C. elegans using conventional NGM (nematode growth medium), and investigation of the possible underlying mechanisms of lifespan extension by ERW using various long-lived genetic mutants.

\section{METHODS \\ C. elegans strains and growth conditions}

The N2 CGCb strain, which is the wild type strain provided by the Caenorhabditis Genetics Center at University of Minnesota was used as control strain in this study. age-1 ( $x \times 546)$ and eat-2 (ad465) are mutant strains whose lifespan is extended through reduced insulin/IGF-1-like signal and decreased food intake, respectively (Friedman and Johnson, 1988; Lakowski and Hekimi, 1998). Worms were cultured at $20{ }^{\circ} \mathrm{C}$ on NGM agar plates (1.7\% agar, $2.5 \mathrm{mg} / \mathrm{mL}$ peptone, $25 \mathrm{mM} \mathrm{NaCl}, 50$ $\mathrm{mM} \mathrm{KH}_{2} \mathrm{PO}_{4} \mathrm{pH6.0,5} \mu \mathrm{g} / \mathrm{mL}$ cholesterol, $1 \mathrm{mM} \mathrm{CaCl}_{2}$, and $1 \mathrm{mM} \mathrm{MgSO}_{4}$ ) seeded with Escherichia coli OP50 as the food source. Age-synchronized hermaphrodites were established by permitting five young adult (L4) worms to lay eggs on a fresh NGM plate for four hours at $20^{\circ} \mathrm{C}$. Liquid NGM was prepared the same as the NGM agar plates without adding agar and sterilized with a $0.2 \mu \mathrm{m}$ cellulose acetate filter (Advantec, Japan). All adult worms were removed from the plate, and the eggs were incubated at $20^{\circ} \mathrm{C}$ until the L4 larval stage.

Preparation of ERW

ERW containing $\mathrm{NaCl}$ was produced with an electrolysis device (208X120X90 mm, 650 g, DoDream, Republic of Korea). The $\mathrm{NaCl}$ concentration of the ERW was $0.08 \%$, the $\mathrm{pH}$ was $10-$ 11 , and the oxidation-reduction potential was $-700 \mathrm{mV}$. The $\mathrm{pH}$ and oxidation-reduction potential values were measured using a $\mathrm{pH} /$ oxidation-reduction potential meter.

\section{Lifespan assay}

Sixty age-synchronized three-day-old worms grown in NGM agar plates with OP50 were transferred to liquid NGM to test the effect of ERW on lifespan. 5-Fluoro-2'-deoxyuridine (SigmaAldrich, USA) was added to the liquid NGM to block progeny development. Worms were transferred to fresh liquid NGM every day during the first four days. After four days, worms were transferred to fresh media every two days until all worms died. Live worms were transferred to a fresh plate, and mortality was scored. Worms that did not respond to any mechanical stimuli were regarded as dead. N2 worms grown in NGM prepared with distilled water were the control group. The survival of the control group was compared with that of the experimental group that were worms grown in NGM prepared with ERW.

\section{Fertility assay}

Age-synchronized worms were grown in liquid NGM with OP50 for two days at $20{ }^{\circ} \mathrm{C}$. A single young adult worm was transferred to new liquid NGM and allowed to lay eggs at 20 ${ }^{\circ} \mathrm{C}$ for 24 hours. The next day, the adult worm was transferred to fresh liquid NGM, and media containing eggs were incubated at $20{ }^{\circ} \mathrm{C}$ for an additional two days. The number of hatched worms was recorded after two days. This process was repeated until no hatched worms were found in the media. The control and experimental groups were worms cultured in NGM prepared with distilled water and ERW, respectively.

Resistance to oxidative stress

To induce oxidative stress in worms, $20 \mathrm{mM}$ paraquat (methyl viologen dichloride hydrate) was added to the liquid NGM. Sixty age-synchronous three-day-old hermaphrodite worms were used in each group. Dead worms were counted three times per day until all worms were dead. N2 and mutant worms grown in NGM prepared with distilled water were the control groups and worms grown in NGM prepared with ERW were the experimental groups.

\section{Statistical analysis}

For the statistical analysis of the lifespan and resistance to oxidative stress assays, we performed the log-rank test (Peto and Peto, 1972). The log-rank test is a non-parametric MantelCox test and one of most widely used statistical methods for the comparison of survival and calculation of mean survival time between control and experimental groups. We measured mean and maximum lifespan and tested statistical significance. For statistical significance of the fertility assay we used a standard two-tailed Student t-test.

RNAi assay

The daf-16 RNAi clone was obtained from the Ahringer RNAi library (Kamath et al., 2003) and verified by sequencing. The Ahringer RNAi library contains genome-wide RNAi clones that can be used for C. elegans gene knockdown. PCR-amplified sequences for each gene were cloned in L4440 vector (Kamath et al., 2003). Five L4 / young adult worms were transferred to a fresh NGM plate containing $100 \mu \mathrm{g} / \mathrm{mL}$ ampicillin, $12.5 \mu \mathrm{g}$ / mL tetracycline, $0.4 \mathrm{mM}$ IPTG and $0.5 \mathrm{mg}$ / mL 5-fluoro-2'deoxyuridine, and spotted with bacteria expressing doublestranded RNA from an empty vector or daf-16. After laying eggs for four hours, all five adult worms were removed. Sixty young adults were selected three days after hatching and were transferred to a fresh plate with double-stranded RNAexpressing bacteria. 
RESULTS

Lifespan of C. elegans was increased by ERW

ERW is generally known for its strong anti-oxidant activity. A study showed that worms grown in ERW lived longer than worms grown in distilled water. However, the effect of ERW in conventional NGM had not yet been studied. To examine the effect of ERW on the lifespan of C. elegans grown in NGM, we compared the lifespan of wild-type N2 worms grown in liquid NGM prepared with distilled water and ERW. Worms grown in liquid NGM prepared with ERW lived significantly longer than worms grown in liquid NGM prepared with distilled water (Fig. 1). The mean lifespan of worms increased from 18.3 days to 21.5 days $(17.3 \%$ increase, $\mathrm{p}=0.004)$, and maximum lifespan was extended by up to 4 days (29 to 33 days). A replicative experiment showed similar results: mean lifespans were 16.7 and 19.6 days and maximum lifespans were 28 and 31 days in distilled water and ERW, respectively (17.5\% increase of mean lifespan; $\mathrm{p}=0.007$ ).

\section{The effect of ERW on reproduction of C. elegans}

Many genetic or environmental interventions that extend lifespan in C. elegans accompany a trade-off of reduced reproduction (Hughes et al., 2007; Larsen et al., 1995). To address whether the longevity phenotype by ERW treatment induces reduced progeny production, we examined the time-course distribution of the number of progeny produced and calculated the total number of progeny produced during the gravid period of worms grown in NGM prepared with ERW. As shown in Figure 2A, worms treated with ERW at day five, seven, and eight produced more progeny compared to the control. A comparison of the total number of progeny during the entire gravid period revealed that ERW conferred increased fertility. Total numbers of progeny were $137.1 \pm 9.2$ in distilled water-medium and $162.7 \pm 10.8$ in ERW-medium (mean \pm SEM) (Fig 2B).

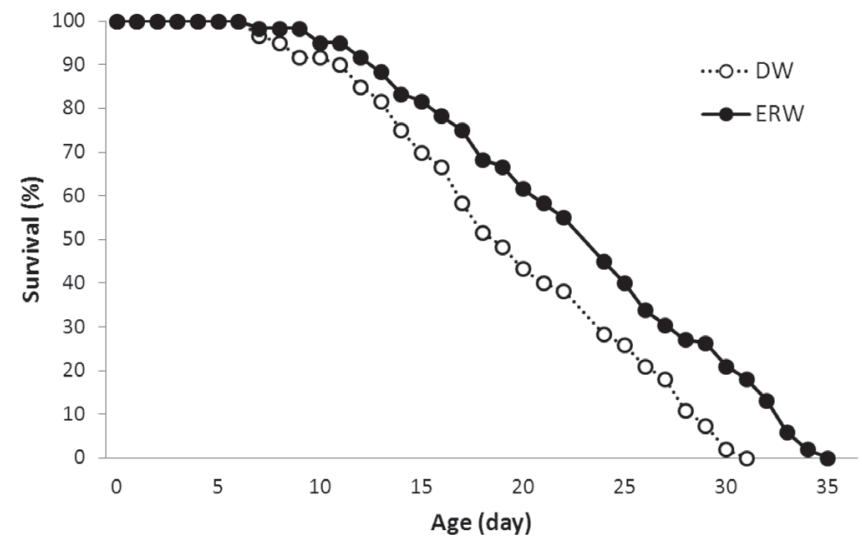

Figure 1: ERW significantly increased both mean and maximum lifespan of Caenorhabditis elegans. Worms grown in liquid NGM prepared with ERW lived longer than worms grown in liquid NGM prepared with distilled water. Mean lifespan of worms increased up to $17.3 \%$ following ERW treatment. Mean lifespans of animals grown in distilled water- and ERW-medium were 18.3 and 21.5 days, respectively $(P=0.004)$. $Y$ axis indicates $\%$ survival, which was calculated as (number of worms survived on each day/total number of worms $) \times 100$. ERW $=$ electrolyzed reduced water.
Lifespan extension by ERW specifically involves the insulin/IGF-1-like signal

We next examined the effect of ERW on long-lived mutants to identify the possible underlying mechanism of longevity by ERW. The age-1 mutant shows increased lifespan due to a reduced insulin/IGF-1-like signal and the eat-2 mutant is a widely-used genetic model of dietary restriction (DR) in C. elegans (Friedman and Johnson, 1988; Lakowski and Hekimi, 1998). These two mechanisms have been demonstrated to extend the lifespan in various organisms (Brown-borg et al., 1996; Greer and Brunet, 2009; Kaeberlein et al., 2005; Kapahi et al., 2004; Masoro, 2005). The lifespan assay revealed that the lifespan of age-1 ( $x \times 546)$ was not further extended by ERW, whereas both mean and maximum lifespan of eat-2 (ad465) significantly increased following ERW treatment (Fig. 3). Mean and maximum lifespan of age-1 ( $h \times 546)$ were 23.0 and 32 days in distilled water and 22.3 and 33 days in ERW, respectively ( $p$ $=0.924)$. Mean and maximum lifespan in eat-2 (ad465) increased from 21.4 and 31 days to 24.9 and 34 days, respectively, following the ERW treatment $(\mathrm{p}=0.009)$. A replicative experiment showed similar results (data not shown).

Resistance to oxidative stress of long-lived mutants grown in ERWmedium

We then asked whether resistance to oxidative stress is altered by ERW in long-lived mutants. Paraquat $(20 \mathrm{mM})$ was used to induce oxidative stress in vivo. As shown in Table 1, age-1 (hx546) and eat-2 (ad465) were more resistant to oxidative stress than wild-type N2 $(\mathrm{p}<0.05)$. Mean survival times in N2 worms after paraquat treatment were 52.6 and 68.8 hours in distilled waterand ERW-treated worms, respectively $(\mathrm{p}=0.002)$. Resistance to oxidative stress also increased by ERW in eat-2 (ad465) from 79.6 hours in distilled water to 92.6 hours with ERW $(\mathrm{p}=0.002)$ and from 64.5 to 80.4 hours $(\mathrm{p}=0.018)$ in replicative experiments. In contrast, no significant change in resistance to oxidative stress by ERW was observed in age-1 ( $h x 546)$ (Table I).

\section{TABLE I}

The effect of ERW on resistance to oxidative stress in long-lived $C$. elegans mutants

\begin{tabular}{llcc}
\hline \multicolumn{1}{c}{ Strain } & \multicolumn{1}{c}{ Medium } & $\begin{array}{c}\text { Mean survival time } \\
\text { (hours) }\end{array}$ & p-value \\
\hline N2 & Distilled water & 52.6 & \\
& ERW & 68.8 & 0.002 \\
age-1 (hx546) & Distilled water & 66.5 & \\
& ERW & 75.3 & 0.169 \\
& Distilled water & 90.4 & \\
& ERW & 98.9 & 0.162 \\
eat-2 (ad465) & Distilled water & 76.9 & \\
& ERW & 92.6 & 0.002 \\
& Distilled water & 64.5 & \\
& ERW & 80.4 & 0.018 \\
\hline
\end{tabular}

Liquid NGM was prepared with distilled water or electrolyzed-reduced water (ERW) as indicated. Data are expressed as mean survival time after treating worms with $20 \mathrm{mM}$ paraquat. The $\mathrm{p}$-value was calculated using the long-rank test 


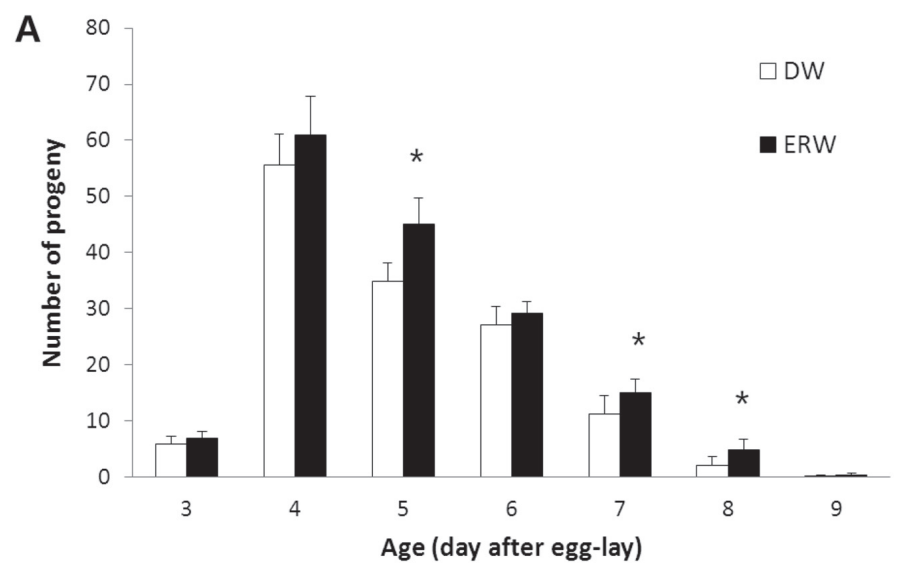

B

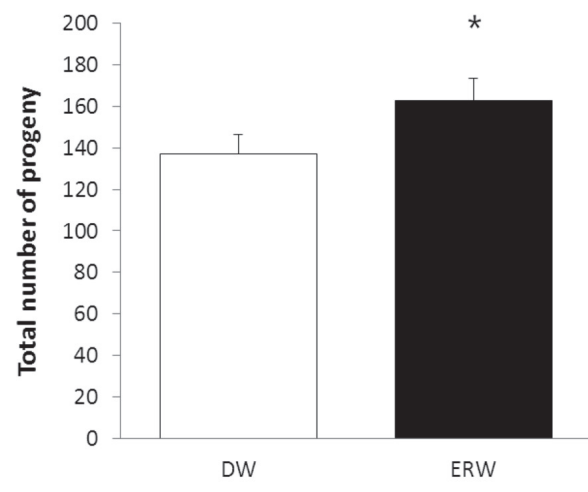

Figure 2: The effect of ERW on Caenorhabditis elegans fertility. Age-synchronized wild type N2 worms were transferred to fresh media every day, and the progeny were counted after 2 days. (A) Time-course distribution of the number of progeny produced. Worms grown in ERW produced more progeny compared to those in distilled water. The $Y$ axis indicates the number of progeny produced on each day. (B) The total number of progeny produced also increased significantly following ERW treatment: 137.1 \pm 9.2 in distilled water and $162.7 \pm 10.8$ in ERW. The $Y$ axis indicates the average total number of progeny produced during gravid period. Data are mean \pm SEM of the number of progeny produced by 10 individual worms. ${ }^{*} \mathrm{p}<0.05$. ERW $=$ electrolyzed reduced water.
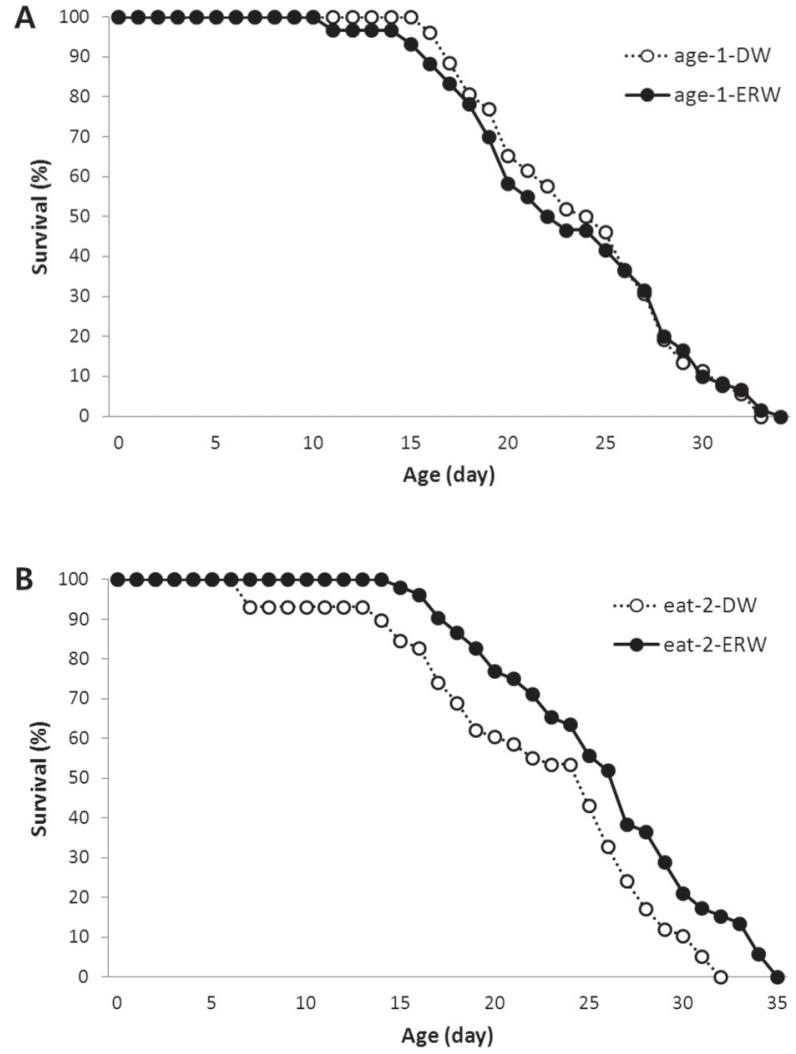

Figure 3: Lifespan-extending effect of ERW overlaps with that of the insulin-IGF-1-like signal. (A) The effect of ERW on the lifespan of age-1 ( $h \times 546)$ was analyzed using a lifespan assay. (B) The lifespans of eat-2 (ad465) grown in NGM prepared with distilled water and ERW were compared. A significant increase in the lifespan of eat-2 (ad465) was observed following ERW treatment. However, ERW failed to extend significantly the lifespan of age1 (hx546). Y axis indicates \% survival, which was calculated as (number of surviving worms each day/total number of worms) $\times 100 . \mathrm{ERW}=$ electrolyzed reduced water.
Lifespan extension by ERW depends on DAF-16

The longevity phenotype with a reduced insulin/IGF-1-like signal requires the transcription factor DAF-16 (Murphy et al., 2003). To elucidate the role of DAF-16 in ERW-induced lifespan extension, we examined the effect of daf-16 RNAi on lifespan of worms grown in distilled water- or ERW-medium. The increased lifespan conferred by ERW was completely abolished by daf-16 RNAi in wild-type N2 worms (Fig. 4A). Mean lifespan increased from 18.2 to 23.0 days by ERW ( $\mathrm{p}<$ 0.001). However, the extended lifespan by ERW was prevented by gene knockdown of daf-16 (mean and maximum lifespans were 15.7 and 21 days, respectively). These findings indicate that the lifespan extension by ERW was dependent on DAF-16, which supports our hypothesis that ERW-induced long lifespan is mediated by a decrease in the insulin/IGF-1 signal. We also observed the same shortened lifespan by daf-16 RNAi in age-1 (hx546) (Fig. 4B). As previously observed, the lifespan of age1 (hx546) was not altered by ERW (mean lifespan of distilled water- and ERW-medium was 22.8 and 22.3 days, respectively). RNAi of daf-16 in age-1 ( $h \times 546)$ significantly reduced both mean and maximum lifespan of worms cultured in distilled water- or ERW-medium.

\section{DISCUSSION}

ERW showed a positive impact on longevity of C. elegans in conventional NGM. Both mean and maximum lifespan were significantly extended in worms grown in NGM prepared with ERW. To our surprise, the reduced fertility which usually accompanies many long-lived mutants was not observed in lifespan extension by ERW. Genetic analysis using mutants and gene knockdown suggest that the cellular mechanisms involved in lifespan extension by ERW overlap with a decreased insulin/IGF-1-like signal and are independent of DR.

ERW is produced by electrolysis of water and has been extensively studied due to its antioxidant activity. C. elegans 
grown in ERW accumulate less ROS induced by the oxidativestress inducer paraquat (Yan et al., 2010). Another study also showed that the conventional NGM C. elegans culture medium prepared with ERW confers increased resistance to various environmental stresses compared to NGM prepared with distilled water (Park et al., 2012). A previous study showed that water medium prepared with ERW extended mean lifespan of C. elegans (11-41\%), but failed to increase maximum lifespan (Yan et al., 2010; Yan et al., 2011). No significant extension of either mean or maximum lifespan by ERW is observed when S-basal medium is used in the lifespan assay (Yan et al., 2010). In the present study, we employed liquid NGM instead of water or S-basal medium to examine the effect of ERW on lifespan, since NGM is the standard culture medium for the C. elegans lifespan assay. A comparison of survival between worms in liquid NGM prepared with distilled water and ERW revealed that ERW extended both mean and maximum lifespan of C. elegans significantly.

Multicellular organisms allocate their resources between growth and maintenance of soma and reproduction in response to environmental changes, and a reduced total number of progeny is observed in many lifespan-extended mutants of
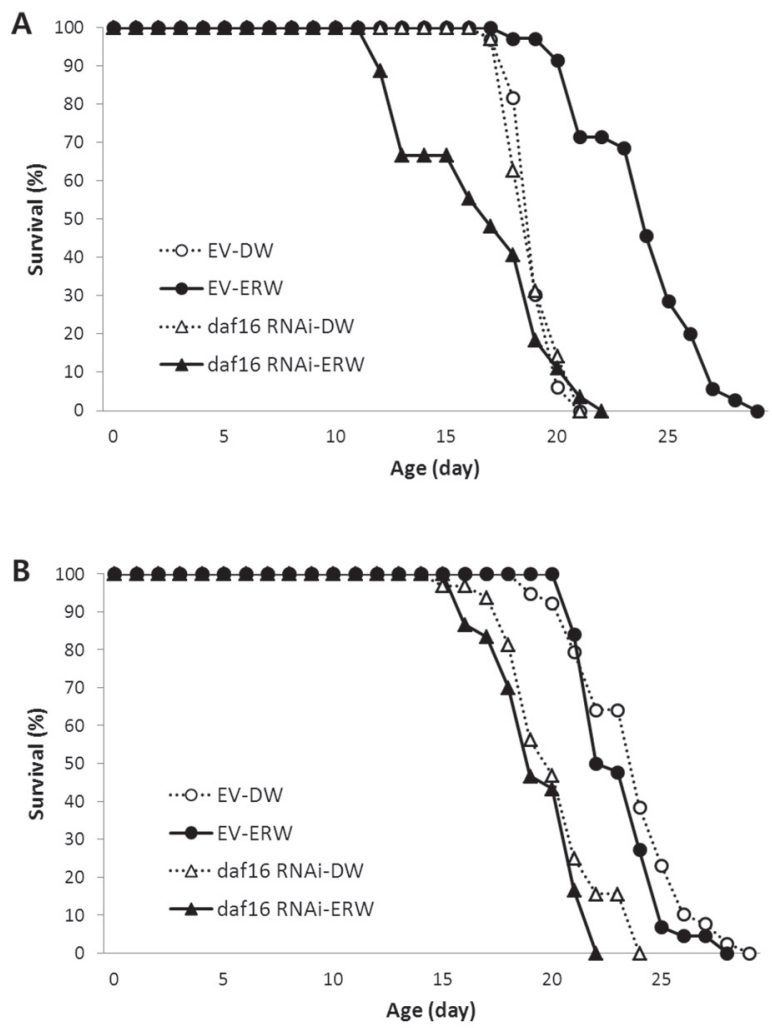

Figure 4: RNAi of daf-16 abolishes the lifespan-extending effect of ERW. (A) The lifespan of wild-type N2 was markedly extended by ERW. However, extended lifespan was completely prevented by daf-16 gene knockdown. (B) No additional increase in lifespan following ERW treatment was observed in age- 1 ( $h \times 546)$, and daf-16 RNAi blocked the lifespan-extending effect of the age- 1 mutant or ERW treatment. The $\mathrm{Y}$ axis indicates \% survival, which was calculated as (number of worms survived on each day/total number of worms) $\times 100$. ERW = electrolyzed reduced water. daf$16=$ RNAi clone expressing double-stranded RNA of daf- 16 gene.
C. elegans (Hughes et al., 2007; Larsen et al., 1995). A slight extension of the reproductive period is also observed in a few long-lived strains (Gems et al., 1998). In addition, the ablation of germ cells increases lifespan and a DR, which extends lifespan in various organisms, delays the reproductive period in C. elegans (Crawford et al., 2007; Hsin and Kenyon, 1999). The number of progeny produced by worms grown in NGM prepared with distilled water and ERW was compared to examine the effect of ERW on reproduction. Unlike other longlived mutants, the longevity phenotype conferred by ERW did not accompany a reduced reproduction phenotype. To our surprise, the total number of progeny was rather moderately increased. These results indicate that ERW is able to extend lifespan without a cost of reduced fertility and might be beneficial not only to extend lifespan, but also for the health span of C. elegans.

The two most studied lifespan-extending mechanisms in C. elegans are a reduced insulin/IGF-1-like signal and DR. The first longevity mutant in C. elegans was age-1, which encodes a PI3 kinase involved in insulin/IGF-1-like signaling. Mutations in other factors mediating the insulin/IGF-1-like signal, such as daf-2 and akt-1/akt-2, also extend survival up to 300\% (Gems et al., 1998; Johnson, 1990; Kenyon et al., 1993). DR is the only intervention that increases lifespan in nearly all species tested (Masoro, 2005). In the present study, the lifespan of eat-2, the genetic DR mutant, was further extended by ERW. However, no additional increase in lifespan was observed when age-1 mutants were grown in NGM prepared with ERW. The longevity phenotype with the reduced insulin/IGF-1-like signal is mediated through the FOXO-family transcription factor DAF16 (Tatar et al., 2003). The lifespan extension by daf-2 RNAi is completely opposed by daf-2 and daf-16 double RNAi (Murphy et al., 2003). Here, we observed that the lifespan-extending effect of ERW was inhibited by daf-16 RNAi. We also observed increased resistance to oxidative stress in age-1 and eat-2 compared to wild type N2. Interestingly, the resistance to oxidative stress in age-1 was not further increased by ERW, whereas wild-type N2 and eat-2 worms displayed increased resistance to oxidative stress following ERW treatment. These results suggest that both insulin/IGF-1-like signal and ERW may modulate longevity through increased resistance to oxidative stress. Based on these findings, we conclude that ERW confers the longevity phenotype by reducing the insulin/IGF-1-like signal in C. elegans and suggest that there should be overlapping mechanisms between the insulin/IGF-1-like signal and ERW treatment.

It is still unknown whether supplementation of antioxidants can retard the aging process and extend lifespan. Our findings that ERW acted as a strong ROS scavenger and extended lifespan with increased resistance to oxidative stress and no reduction in fertility support the free radical theory of aging. Further studies on the underlying mechanisms involved in ERW, the effect on age-related diseases, and the influence on lifespan of other species will strengthen our understanding of the effect of ERW on aging and provide a possible clinical application for ERW.

\section{ACKNOWLEDGEMENTS}

This research was supported by the Basic Science Research Program through the National Research Foundation of Korea (NRF) funded by the Ministry of Education, Science and Technology (2010-0022429). 


\section{REFERENCES}

BECKMAN KB, AMES BN (1998) The free radical theory of aging matures. Physiol Rev 78:547-581.

BROWN-BORG HM, BORG KE, MELISKA CI, BARTKE A (1996) Dwarf mice and the ageing process. Nature 384:33.

CRAWFORD D, LIBINA N, KENYON C (2007) Caenorhabditis elegans integrates food and reproductive signals in lifespan determination. Aging Cell 6:715-721.

FRIEDMAN DB, JOHNSON TE (1988) A mutation in the age-1 gene in Caenorhabditis elegans lengthens life and reduces hermaphrodite fertility. Genetics 118:75-86.

GEMS D, SUTTON AJ, SUNDERMEYER ML, ALBERT PS, KING KV, EDGLEY ML, LARSEN PL, RIDDLE DL (1998) Two pleiotropic classes of daf-2 mutation affect larval arrest, adult behavior, reproduction and longevity in Caenorhabditis elegans. Genetics 150:129-155.

GREER EL, BRUNET A (2009) Different dietary restriction regimens extend lifespan by both independent and overlapping genetic pathways in C. elegans. Aging Cell 8:113-127.

HSIN H, KENYON C (1999) Signals from the reproductive system regulate the lifespan of C. elegans. Nature 399:362-366.

HUGHES SE, EVASON K, XIONG C, KORNFELD K (2007) Genetic and pharmacological factors that influence reproductive aging in nematodes. PLoS Genet 3:e25.

JOHNSON TE (1990) Increased life-span of age-1 mutants in Caenorhabditis elegans and lower Gompertz rate of aging. Science 249:908-912.

KAEBERLEIN M, POWERS RW 3RD, STEFFEN KK, WESTMAN EA, HU D, DANG N, KERR EO, KIRKLAND KT, FIELDS S, KENNEDY BK (2005) Regulation of yeast replicative life span by TOR and Sch9 in response to nutrients. Science 310:1193-1196.

KAMATH RS, FRASER AG, DONG Y, POULIN G, DURBIN R, GOTTA M, KANAPIN A, LE BOT N, MORENO S, SOHRMANN M, WELCHMAN DP, ZIPPERLEN P, AHRINGER J (2003) Systematic functional analysis of the Caenorhabditis elegans genome using RNAi. Nature 421:231-237.

KAPAHI P, ZID BM, HARPER T, KOSLOVER D, SAPIN V, BENZER S (2004) Regulation of lifespan in Drosophila by modulation of genes in the TOR signaling pathway. Curr Biol 14:885-890.

KENYON C, CHANG J, GENSCH E, RUDNER A, TABTIANG R (1993) A C. elegans mutant that lives twice as long as wild type. Nature 366:461-464.

KIM C, HUNG YC, BRACKETT RE (2000) Efficacy of electrolyzed oxidizing (EO) and chemically modified water on different types of foodborne pathogens. Int J Food Microbiol 61:199-207.

LAKOWSKI B, HEKIMI S (1998) The genetics of caloric restriction in Caenorhabditis elegans. Proc Natl Acad Sci U S A 95:13091-13096.

LAKSHMANAN AP, WATANABE $K$, THANDAVARAYAN RA, SARI FR, MEILEI H, SOETIKNO V, ARUMUGAM S, GIRIDHARAN VV, SUZUKI K, KODAMA M (2011) Curcumin attenuates hyperglycaemia-mediated AMPK activation and oxidative stress in cerebrum of streptozotocininduced diabetic rat. Free Radic Res 45:788-795.

LARSEN PL, ALBERT PS, RIDDLE DL (1995) Genes that regulate both development and longevity in Caenorhabditis elegans. Genetics 139:1567-1583.

LEE MY, KIM YK, RYOO KK, LEE YB, PARK EJ (2006) Electrolyzed-reduced water protects against oxidative damage to DNA, RNA, and protein. Appl Biochem Biotechnol 135:133-144.

LEE KJ, JIN D, CHANG BS, TENG YC, KIM DH (2009) The immunological effects of electrolyzed reduced water on the Echinostoma hortense infection in C57BL/ 6 mice. Biol Pharm bull 32:456-462.
LI Y, HAMASAKI T, NAKAMICHI N, KASHIWAGI T, KOMATSU T, YE J, TERUYA K, ABE M, YAN H, KINJO T, KABAYAMA S, KAWAMURA M, SHIRAHATA S (2001) Suppressive effects of electrolyzed reduced water on alloxan-induced apoptosis and type 1 diabetes mellitus. Cytotechnology 63:119-131.

MASORO EJ (2005) Overview of caloric restriction and ageing. Mech Ageing Dev 126:913-922.

MURPHY CT, MCCARROLL SA, BARGMANN CI, FRASER A, KAMATH RS, AHRINGER J, LI H, KENYON C (2003) Genes that act downstream of DAF-16 to influence the lifespan of Caenorhabditis elegans. Nature 424:277-283.

PETO R, PETO J (1972) Asymptotically efficient rank invarient test procedures. J Royal Stac Soc Ser A 135:185-207.

PARK H, HUNG YC, BRACKETT RE (2002a) Antimicrobial effect of electrolyzed water for inactivating Campylobacter jejuni during poultry washing. Int J Food Microbiol 72:77-83.

PARK H, HUNG YC, KIM C (2002b) Effectiveness of electrolyzed water as a sanitizer for treating different surfaces. J Food Prot 65:1276-1280.

PARK SK, PAGE GP, KIM K, ALLISON DB, MEYDANI M, WEINDRUCH R, PROLLA TA (2008) alpha- and gamma-Tocopherol prevent agerelated transcriptional alterations in the heart and brain of mice. J Nutr 138:1010-1018

PARK SK, KIM K, PAGE GP, ALLISON DB, WEINDRUCH R, PROLLA TA (2009) Gene expression profiling of aging in multiple mouse strains: identification of aging biomarkers and impact of dietary antioxidants. Aging Cell 8:484-495.

PARK SK, KIM JJ, YU AR, LEE MY, PARK SK (2012) Electrolyzed-reduced water confers increased resistance to environmental stresses. Mol Cell Toxicol 8:241-247.

SHIRAHATA S, KABAYAMA S, NAKANO M, MIURA T, KUSUMOTO K, GOTOH M, HAYASHI H, OTSUBO K, MORISAWA S, KATAKURA Y (1997) Electrolyzed-reduced water scavenges active oxygen species and protects DNA from oxidative damage. Biochem Biophys Res Commun 234:269-274.

SOHAL RS, WEINDRUCH R (1996) Oxidative stress, caloric restriction, and aging. Science 273:59-63.

SREEJAYAN N, RAO MN (1996) Free radical scavenging activity of curcuminoids. Arzneimittelforschung 46:169-171.

STORZ G, IMLAY JA (1999) Oxidative stress. Curr Opin Microbiol 2:188-194.

TATAR M, BARTKE A, ANTEBI A (2003) The endocrine regulation of aging by insulin-like signals. Science 299:1346-1351.

THE C. ELEGANS SEQUENCING CONSORTIUM (1998) Genome sequence of the nematode C. elegans: a platform for investigating biology. Science 282: 2012-2018.

VENKITANARAYANAN KS, EZEIKE GO, HUNG YC, DOYLE MP (1999) Inactivation of Escherichia coli O157:H7 and Listeria monocytogenes on plastic kitchen cutting boards by electrolyzed oxidizing water. J Food Prot 62:857-860.

WOOD WB (1988) The nematode Caenorhabditis elegans. Cold Spring Harbor, USA: Cold Spring Harbor Laboratory Press. pp:1-16.

WOOD JG, ROGINA B, LAVU S, HOWITZ $K$, HELFAND SL, TATAR M, SINCLAIR D (2004) Sirtuin activators mimic caloric restriction and delay ageing in metazoans. Nature 430:686-689.

YAN H, TIAN H, KINIO T, HAMASAKI T, TOMIMATSU $K$, NAKAMICHI N, TERUYA K, KABAYAMA S, SHIRAHATA S (2010) Extension of the lifespan of Caenorhabditis elegans by the use of electrolyzed reduced water. Biosci Biotechnol Biochem 74:2011-2015.

YAN H, KINJO T, TIAN H, HAMASAKI T, TERUYA K, KABAYAMA S, SHIRAHATA S (2011) Mechanism of the lifespan extension of Caenorhabditis elegans by electrolyzed reduced water--participation of Pt nanoparticles. Biosci Biotechnol Biochem 75:1295-1299. 